\title{
Cool Dynamics: Tapping the Potential of Low Temperature ESEM
}

\author{
D.Waller,* D.J. Stokes, ${ }^{*}$ J-Y Mugnier** \& A.M. Donald* \\ *Biological \& Soft Systems, Cavendish Laboratory, Madingley Road, Cambridge, CB3 0HE, UK \\ ** Unilever R\&D, Colworth House, Sharnbrook, Bedford, MK44 1LQ, U.K.
}

With the ability to view uncoated samples without the effects of charge build up, low vacuum and environmental scanning electron microscopy have expanded the field of electron microscopy and associated dynamic experiments. Some examples include; the swelling behaviour of fibrils [1], strain deformation in polymer gels [2] and the interaction between water and carbon nanotubes [3]. One aspect that has received little attention is the potential for experiments at sub-zero temperatures, for example; in the range -80 to $0^{\circ} \mathrm{C}$. Figure 1 depicts some thermal regimes available for (E)SEM experiments in relation to the SVP curve for water.

We have been developing methodologies that enable observation of in situ low temperature dynamic experiments such as; freeze-drying, thermal cycling, ice crystal nucleation, growth and morphology, and phase transitions. Maintaining a stable frozen-hydrated sample requires the addition of water vapour at temperatures above approximately $-80^{\circ} \mathrm{C}$. Previous work has demonstrated the use of low vacuum mode, where water vapour and another gas were used as a means of thermodynamic stability and imaging, respectively [4-6].

We used a Gatan Alto 2100 cryosystem in an FEI XL30 ESEM FEG with nitrogen as the imaging gas. In order to prevent any added water vapour precipitating out as ice, cold surfaces such as the cryo stage and coolant gas inlet and outlet pipes were thermally lagged. Using this technique we were able to maintain a stable, frozen-hydrated sample up to $-60{ }^{\circ} \mathrm{C}$.

As the required water vapour pressure increases, we find that even lagging is insufficient to prevent ice precipitation. In order to improve our control over the stability of the specimen, an alternative approach is needed for experiments at temperatures above about $-60^{\circ} \mathrm{C}$.

Our new technique involves ensuring the sample is the coldest surface. This involves two additions to our apparatus; a Peltier module and a cartridge heater. The cartridge heater is used to control the temperature of the coolant gas before it enters the chamber. By using a Peltier chip in the cryotransfer module, we can independently control the sample temperature to a few degrees below the cryostage temperature. This approach enables us to control the state of the specimen across the entire temperature range of interest.

We have also found that the presence of an imaging gas, such as nitrogen, reduces the rate of any sublimation, enabling good control for freeze drying and etching for example. This is demonstrated in Figure 2.

Thus, the ability to control both the state of the specimen and its environment gives great scope for the observation of dynamic processes at temperatures not usually accessible in SEM 


\section{References}

[1] L.M. Jenkins et al., Scanning 19 (1997) 92

[2] R. Rizzieri et al., Polymer 44 (2003) 5927

[3] M.P. Rossi et al. , Nano Lett. 4 (5) (2004) 989

[4] P. Meredith et al., J. Pharm. Sci. 85(6) (1996)631

[5] A.L. Fletcher, Phd thesis (1997) Uni. Cambridge.

[6] D.J. Stokes et al., J. Microscopy 213(2) (2004) 198

[7] The author gratefully acknowledges Unilever for their generous support.

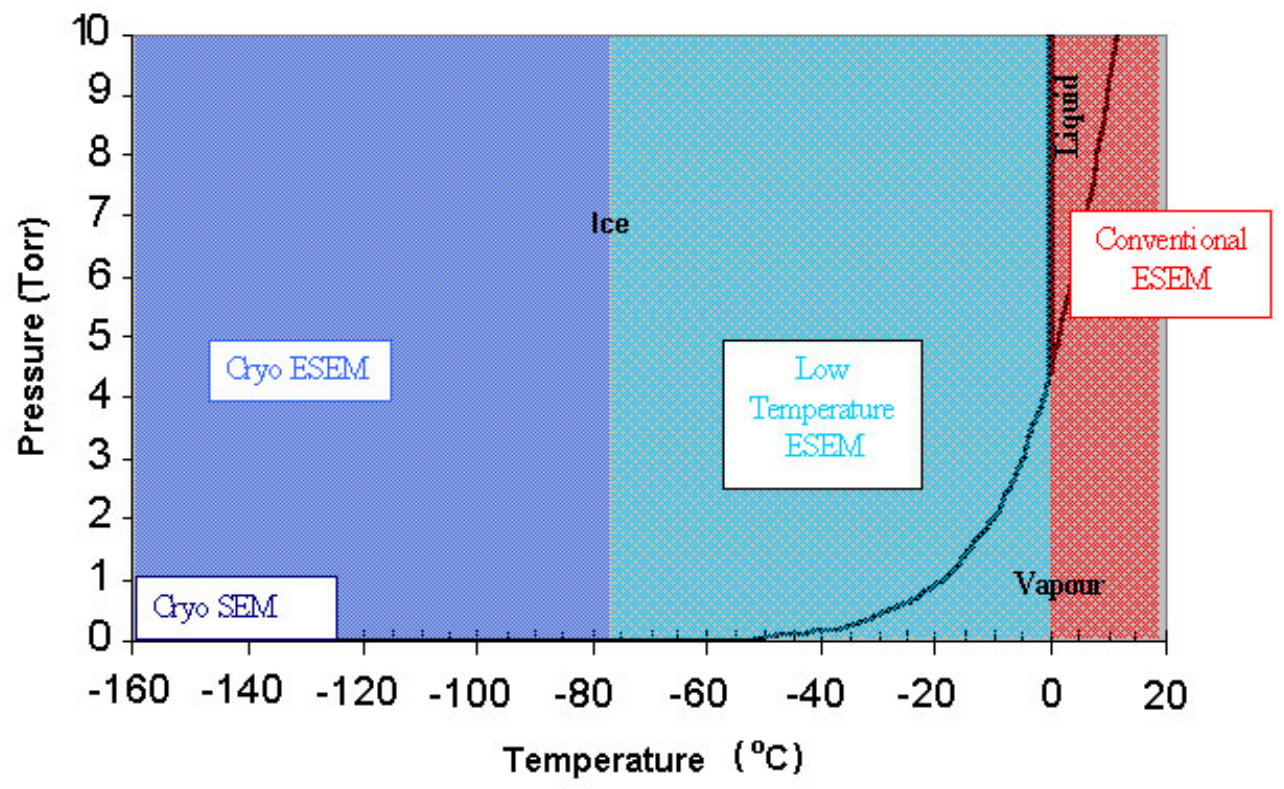

Figure 1. SVP curve for water and sample temperatures for different techniques

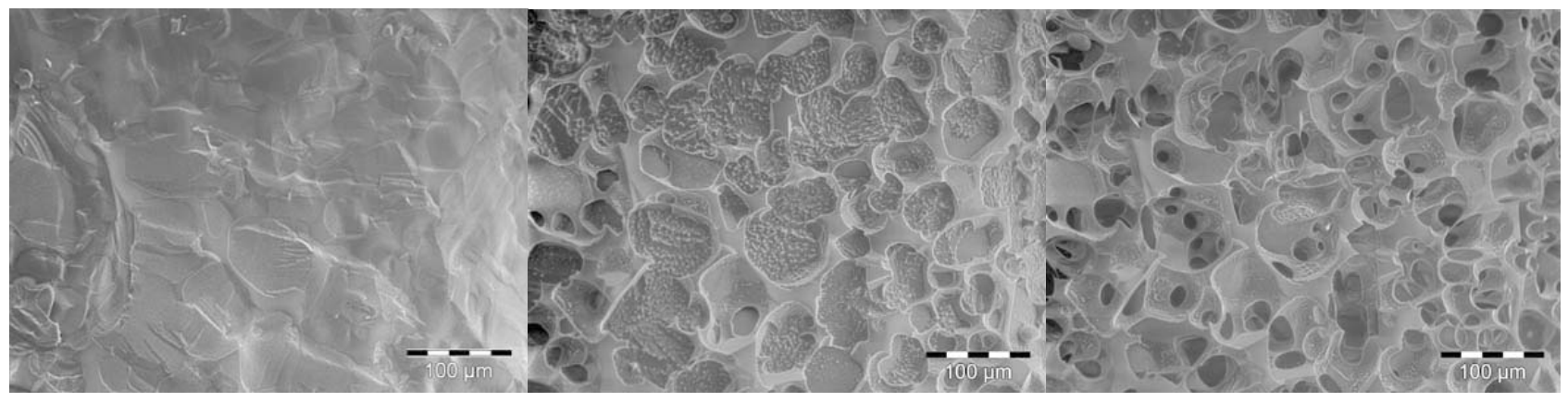

Figure 2. Controlled sublimation of a sucrose and ice mix. The initially featureless surface was etched over a period of 20 minutes at $-75^{\circ} \mathrm{C}$, leaving a highly porous, interconnected foam structure 